\title{
Abscission and Kernel Quality of Almond Fruit Inoculated with the Shot Hole Pathogen Wilsonomyces carpophilus
}

\author{
Beth L. Teviotdale, Extension Plant Pathologist, Nancy Goodell, Staff Research Associate, and Dennis Harper, \\ Staff Research Associate, University of California Davis/Kearney Agricultural Center, Parlier 93648
}

\begin{abstract}
Teviotdale, B. L., Goodell, N., and Harper, D. 1997. Abscission and kernel quality of almond fruit inoculated with the shot hole pathogen Wilsonomyces carpophilus. Plant Dis. 81:14541458.

Fruit of almond cultivar Mission were aerosol-spray inoculated with aqueous suspensions containing $10^{3}, 10^{4}$, or $10^{5}$ conidia/ml of Wilsonomyces carpophilus beginning when fruit emerged from the hypanthium and continuing at approximately weekly intervals until they reached full size and embryo development had begun. There was a linear increase in percent fruit abscission $(P=0.001)$ and average number lesions per fruit $(P=0.007)$ with increasing inoculum concentration. Percent fruit abscission decreased with increasing fruit length (maturity) at inoculation $(P=0.001)$. Lesions were most abundant when mid-size fruit were inoculated. The kernel quality of infected and healthy fruit of cultivar Carmel were compared in 1994 and 1995. Mid- to full-size fruit were inoculated one or three times with $10^{5}$ conidia/ml of W. carpophilus. Kernels from inoculated fruit were shorter than those from uninoculated fruit $(P=0.007)$, but kernel weight did not differ. Fewer than $2.5 \%$ of kernels in 1994 and none in 1995 were covered with gum deposits or incompletely filled.
\end{abstract}

Additional keywords: Prunus dulcis

Shot hole disease of stone fruit trees is caused by the fungus Wilsonomyces carpophilus (Lev.) Adaskaveg, Ogawa, \& Butler. The fungus produces lesions on leaves, fruit, flowers, and succulent shoots. Leaf infection, especially of immature leaves, leads to defoliation, which is the most serious aspect of shot hole disease on almond (Prunus dulcis (Mill.) D.A. Webb). Severe defoliation during early fruit development can cause drop of young fruit, and repeated defoliation weakens trees and reduces yield $(4,5)$. Extended rainfall in spring promotes severe shot hole outbreaks, and growers regularly treat almond trees with fungicides to control the disease $(6,10)$.

Fruit infections produce small, corky, slightly raised lesions that sometimes exude gum. Direct damage to the edible kernel usually is not associated with shot hole fruit infection, although some injury has been reported $(2,4,6,10)$. Occasionally, multiple, coalesced hull infections deform the fruit but the nutmeat appears normal. Multiple lesions clustered along the suture may crack the pericarp, resulting in drying of the embryo (10). Some fruit produce

Corresponding author: Beth L. Teviotdale

E-mail: betht@uckac.edu

Accepted for publication 2 September 1997.

Publication no. D-1997-1027-03R

(C) 1997 The American Phytopathological Society internal gum, and dried gum deposits on the kernel impart a lacquered appearance to the kernel and render it unmarketable. Excessive occurrence of such kernels have been associated with trees having severe shot hole infection, but these are casual observations not supported by experimental evidence.

Several workers have attributed yield loss to shot hole disease $(4,9,10)$. In each case, higher yields and lower disease incidence were recorded from fungicidetreated than from untreated trees. In these studies, yield loss was credited to defoliation because kernel quality was similar in fruit from trees with disparate disease incidence. These conclusions, however, were based on measurements of fruit samples from trees with varying amounts of leaf and fruit infection, and not on evaluation of individual fruit. Furthermore, disease levels on fruit were determined after fruit reached full size, and effects on younger fruit were not measured.

Almond trees finish bloom in early March, and unfertilized fruit grow for about 3 weeks to 5 to $10 \mathrm{~mm}$ in length, then fall from the tree (5). Fertilized fruit continue to lengthen but remain soft until they attain full size at the end of April or early May, when embryo development and shell hardening ensue. A portion of the fertilized fruit are shed in March and April during fruit elongation and before they reach maximum size. Until they begin to yellow and cease to lengthen, fertilized fruit destined to drop prematurely are in- distinguishable from those that will be retained. Defoliation and moisture stress, especially during early fruit growth stages, may cause wrinkling and shriveling of the kernel (5).

Fruit may vary in susceptibility and response to infection at different stages of development. Infected young fruit may fall or produce kernels of lesser quality, and kernel quality may be influenced by the severity of infection. Our objectives were to determine the effects of the stage of fruit development and disease severity on abscission and kernel quality of almond fruit inoculated with $W$. carpophilus.

\section{MATERIALS AND METHODS}

The experiments were conducted in orchards planted with cultivars Mission and Nonpareil or Carmel and Nonpareil, located at the University of California Kearney Agricultural Center in Fresno County, California. Trees were approximately 13 years old, flood-furrow irrigated, and not treated with fungicides.

Inoculum was prepared from an isolate of W. carpophilus obtained from almond and maintained on acidified $(2.5 \mathrm{ml} 25 \%$ lactic acid [vol/vol] per liter of medium) potato dextrose agar (APDA). Cultures were grown at room temperature (20 to $22^{\circ} \mathrm{C}$ ) in the dark for 2 weeks, followed by 1 to 2 weeks under fluorescent laboratory lights. Conidia were collected with sterile deionized water, passed through 3 layers of cheesecloth to remove mycelial fragments, counted with a hemacytometer, and the concentration adjusted with sterile water. Percent germination was determined from 100 conidia in each of two APDA culture plates seeded with $0.1 \mathrm{ml}$ inoculum after incubation at 20 to $22^{\circ} \mathrm{C}$ for $24 \mathrm{~h}$. Germination ranged from 76 to $93 \%$. Inoculum suspensions were stored on ice while in the field.

Inoculum concentration and fruit length. Mission almond fruit were inoculated at approximately weekly intervals beginning as they emerged from the hypanthium until they reached full size (34.8 mm average length) and embryo development began. Sixty fruit in each of four replications were thoroughly wetted with water from a Nalgene aerosol spray bottle (Naljet No. 24330-0200, Nalgene, Rochester, NY). With a similar sprayer, each fruit was inoculated immediately with 0.5 to $1.0 \mathrm{ml}$ (greater volume was required to cover larger fruit) of suspensions contain- 
ing $10^{3}, 10^{4}$, or $10^{5}$ conidia/ml (cpm). Fruit were inoculated once on 26 March, 5, 12, 19, or 26 April 1990, or 18 or 24 March, 8, 16, or 23 April 1993. Uninoculated controls were included on each date and a different tree was used each time.

Inoculated and uninoculated control fruit were kept wet with a fine mist for $48 \mathrm{~h}$ with a system that consisted of standard drip irrigation hose strung through the tree canopy and fitted with fan-jet $340^{\circ}$ misting nozzles (Bowsmith Co., Anaheim, CA) with a capacity of 3.8 liters/h and operated at $3.45 \times 10^{4} \mathrm{~Pa}$. The misting system was operated by an automatic timer (RainMatic 3000, RainMatic Corp., Omaha, NE) that delivered water for $2 \mathrm{~min}$ at 10 -min intervals.

We used fruit length as an indication of fruit age. The average length $(\mathrm{mm})$ of 20 fruit, selected arbitrarily from among inoculated fruit, was measured on each inoculation date to determine fruit length at inoculation. The average fruit length $(\mathrm{mm})$ on each respective inoculation date was $8.6,17.1,24.2,31.3$, and 34.1 in 1990 , and 10.4, 18.0, 27.4, 31.5, and 35.6 in 1993.

Fruit abscission and lesion development were observed on 24 and 13 April 1990 and 1993, respectively, in treatments that had been implemented by those dates. Each fruit was gently tapped, and those that fell were returned to the laboratory and inspected for shot hole infection. Fruit from all inoculation dates for each inoculum concentration were combined and the percentage of fruit having coalesced, discrete, or no visible lesions was determined. Twenty representative fruit from each lesion group were surface disinfested for 3 to $5 \mathrm{~min}$ in a $1: 10$ dilution of $5.25 \%$ sodium hypochlorite, and tissue samples from each were cultured on APDA to confirm the presence of $W$. carpophilus. Final fruit abscission in all treatments was determined from counts of fruit that were firmly attached to the trees on 24 May 1990 and 11 June 1993. Disease severity on 10 (or all that were present if fewer than 10 remained) fruit gathered from each replication was determined on 29 May 1990 and 15 June 1993. Fruit were soaked in water for $5 \mathrm{~min}$ to make the shot hole lesions more visible and easier to count.

Kernel quality. The effect of shot hole infection on kernel quality was examined. Seventy Carmel fruit in each of four replications were inoculated once with a suspension of $10^{5} \mathrm{cpm}$ on 28 March, 4, 13, and 27 April 1994, and 26 March, 6, 14, and 29 April 1995. A different tree was used on each inoculation date. Fruit on a separate tree were inoculated three times, on the last three dates each year, to augment the severity of hull infection. Uninoculated control fruit were included on each inoculation date and with the repeated inoculation treatment. Trees were misted for $48 \mathrm{~h}$ after inoculation as described above. Fruit length was used to represent fruit maturity. The average length $(\mathrm{mm})$ of 20 inoculated fruit selected arbitrarily on each respective inoculation date was $25.4,30.4,33.4$, and 38.3 in 1994 , and 24.7, 29.0, 32.1, and 38.4 in 1995.

Disease severity was evaluated, as described above, on 10 fruit per replication on 23 June 1994 and 6 July 1995. All remaining fruit were harvested on 29 and 26 August 1994 and 1995, respectively. The kernels of 50 fruit per replication were removed and assessed for percentage that had gum deposits (lacquered) or were incompletely filled (shriveled). Shriveled and aborted kernels differ in that part of the former is fully formed and part is withered, whereas aborted kernels are entirely withered. Lacquered and shriveled kernels were replaced with healthy kernels so that 50 healthy kernels were air dried for $72 \mathrm{~h}$ at $65^{\circ} \mathrm{C}$ in a forced air oven, weighed, and measured.

Weather data were collected from a California Irrigation Management Information System station located approximately $0.8 \mathrm{~km}$ from the experimental orchards at the Kearney Agricultural Center.

Experimental design and analysis. There were four replications, each located in one quadrant of a tree, and different trees were used on each inoculation date. Analysis of variance was performed for the experiments from both years combined, using a completely randomized experimental design. The two main plot factors were inoculum concentration and year with fruit inoculation length as a split-plot factor within year. An arcsine transformation was performed on data for percent fruit abscission before analysis of variance. Means were separated by orthogonal contrasts.

\section{RESULTS}

Inoculum concentration and fruit length at inoculation. Fruit abscission was less in 1990 than in $1993(P=0.001)$, decreased linearly with decreasing inoculum concentration $(P=0.001)$, and was greater in inoculated than uninoculated treatments $(P=0.001$; Table 1$)$. As the average fruit length at inoculation increased, percent fruit abscission decreased $(P=0.001)$. Year and inoculum concentration each interacted with fruit length at inoculation. Abscission was greater in 1993 than in 1990 among fruit inoculated at average lengths of 17.5 or $25.8 \mathrm{~mm}$, but was similar among those of other lengths in both years (Fig. 1A). Abscission among fruit inoculated at average lengths of 9.5 , 31.4 , or $34.8 \mathrm{~mm}$ was unaffected by inoculum concentration, whereas abscission among fruit averaging 17.5 or $25.8 \mathrm{~mm}$

Table 1. Effect of inoculum concentration and fruit length at inoculation on infection and abscission of Mission almond fruit inoculated with the shot hole pathogen Wilsonomyces carpophilus, Fresno County, California

\begin{tabular}{|c|c|c|}
\hline & Fruit abscission $(\%)^{\mathrm{a}}$ & Lesions/fruit ${ }^{b}$ \\
\hline \multicolumn{3}{|l|}{ Year } \\
\hline 1990 & 40.5 & 10.5 \\
\hline 1993 & 50.2 & 9.2 \\
\hline \multicolumn{3}{|l|}{ Concentration, conidia/ml } \\
\hline $10^{5}$ & 52.8 & 17.7 \\
\hline $10^{4}$ & 45.9 & 12.7 \\
\hline $10^{3}$ & 43.8 & 7.5 \\
\hline Uninoculated & 38.9 & 1.5 \\
\hline \multicolumn{3}{|c|}{ Average fruit length at inoculation $(\mathrm{mm})^{\mathrm{c}}$} \\
\hline 9.5 & 93.1 & 0.7 \\
\hline 17.5 & 61.9 & 6.0 \\
\hline 25.8 & 36.9 & 24.7 \\
\hline 31.4 & 9.6 & 15.3 \\
\hline 34.8 & 3.4 & 2.7 \\
\hline \multicolumn{3}{|l|}{ Significance of $F, P={ }^{\mathrm{d}}$} \\
\hline Year $(\mathrm{Y})$ & 0.001 & NS \\
\hline Concentration (C) & 0.001 & 0.001 \\
\hline Fruit length at inoculation (L) & 0.001 & 0.001 \\
\hline $\mathrm{Y} \times \mathrm{C}$ & 0.095 & NS \\
\hline $\mathrm{Y} \times \mathrm{L}$ & 0.001 & 0.001 \\
\hline $\mathrm{C} \times \mathrm{L}$ & 0.004 & 0.000 \\
\hline $\mathrm{Y} \times \mathrm{C} \times \mathrm{L}$ & NS & NS \\
\hline \multicolumn{3}{|l|}{ Orthogonal contrasts } \\
\hline \multicolumn{3}{|l|}{ Concentration } \\
\hline Linear & 0.001 & 0.007 \\
\hline Inoculated vs. uninoculated & 0.001 & 0.001 \\
\hline \multicolumn{3}{|l|}{ Fruit length at inoculation } \\
\hline Linear & 0.001 & 0.010 \\
\hline Quadratic & 0.073 & 0.001 \\
\hline
\end{tabular}

a Sixty fruit per replication inoculated once each at approximately weekly intervals during March and April; percent fruit abscission was determined on 24 May 1990 and 11 June 1993.

${ }^{\mathrm{b}}$ Ten fruit per replication observed on 29 May 1990 and 15 June 1993.

${ }^{c}$ The length (mm) of 20 fruit, selected arbitrarily from among inoculated fruit, was measured on each inoculation date.

${ }^{\mathrm{d}}$ Four replications of each treatment. Means are for main effects; NS $=$ not significant, $P>0.100$. 
long at inoculation was greater when inoculated with $10^{5}$ than with $10^{4}$ or $10^{3} \mathrm{cpm}$ (Fig. 1B).

The average number of lesions per fruit in 1990 and 1993 did not differ between years (Table 1). Decreasing inoculum concentration was accompanied by a linear decrease in the average number of lesions per fruit $(P=0.007)$, and more lesions occurred in inoculated than uninoculated treatments $(P=0.001)$. Lesions were more abundant on fruit inoculated when midsized (average 25.8 to $31.4 \mathrm{~mm}$ ) than when smaller or larger $(P=0.001)$. Year and inoculum concentration each interacted with fruit length at inoculation. Lesion numbers on fruit inoculated at average lengths of 9.5 or $34.8 \mathrm{~mm}$ were similar in both years, but differed between years for those inoculated at other lengths (Fig. 2A). Similarly, inoculum concentration did not affect lesion number among the shortest or longest inoculated fruit, but produced varying numbers of lesions on fruit inoculated at intermediate lengths (Fig 2B).
W. carpophilus was isolated from 50 to $90 \%$ of the representative samples collected in April 1990 and 1993. The pathogen was recovered from some young fruit that had not developed typical shot hole lesions, and was more easily cultured from areas of coalesced than discrete lesions. Areas of coalesced lesions were most abundant (40 to 60\%), occasional (less than $10 \%$ ), and absent on fruit inoculated with $10^{5}, 10^{4}$, and $10^{3} \mathrm{cpm}$, respectively. No fruit were deformed.

Kernel quality. Average kernel weight and length were greater in 1994 than in $1995(P=0.002$ and 0.001 , respectively; Table 2). Inoculation did not affect kernel weight, but produced shorter kernels in inoculated than uninoculated fruit $(P=$ 0.007). Fruit length at inoculation did not influence kernel weight or length. Shriveled or lacquered kernels did not exceed $2.5 \%$ in any treatment in 1994 and were not observed in 1995 (data not presented).

Abscission among fruit inoculated on the first date each year was 76.7 and $84.3 \%$, and among uninoculated controls was 52.6 and $68.7 \%$ in 1994 and 1995, respectively. Too few fruit from these first inoculation dates remained to assess fruit quality. Abscission was less than $6.0 \%$ among the other treatments.

There were more lesions per fruit in 1994 than in $1995(P=0.001)$, and more lesions on inoculated than uninoculated fruit $(P=0.001)$. The average number of lesions per fruit decreased as average fruit length at inoculation increased $(P=0.001)$, and more lesions were produced by multiple than single inoculations $(P=0.001)$. An interaction between year and inoculation occurred because the average number of lesions per fruit found in inoculated and uninoculated treatments differed more in 1994 than in 1995 (Fig. 3A). Lesion numbers on uninoculated fruit did not exhibit the linear relationship with fruit inoculation length observed for inoculated fruit, which resulted in an interaction between inoculation and fruit length at inoculation (Fig. 3B).

Weather data. Rainfall during bloom (late February through early March) each
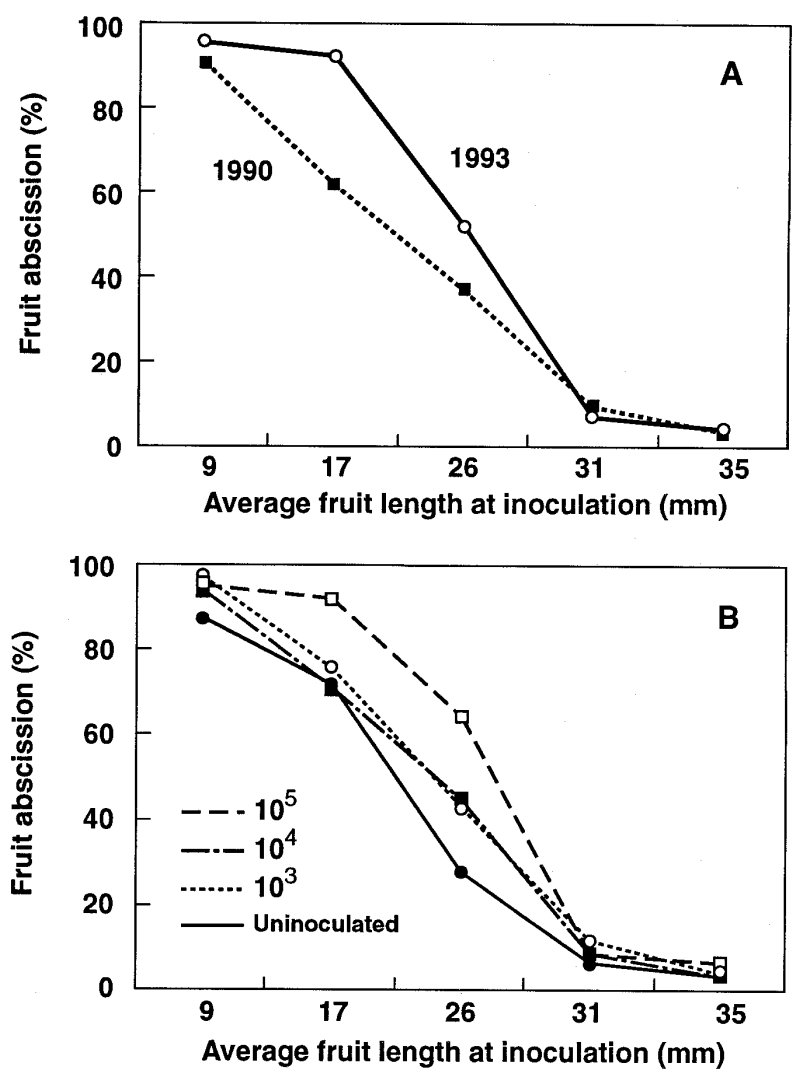

Fig. 1. Interactions of year (A) and inoculum concentration (B) with fruit length at inoculation for percentage abscission of Mission almond fruit inoculated with Wilsonomyces carpophilus, Fresno County, California. Sixty fruit in each of four replications were inoculated during March and April 1990 and 1993 with 0.5 to $1.0 \mathrm{ml}$ (greater volume was required to cover larger fruit) of aqueous suspensions of $10^{3}, 10^{4}$, or $10^{5}$ conidia/ml, or left uninoculated. Average fruit length at inoculation was determined from 20 fruit selected arbitrarily from among inoculated fruit and measured on each inoculation date. Percent abscission was determined on 24 May 1990 and 11 June 1993.
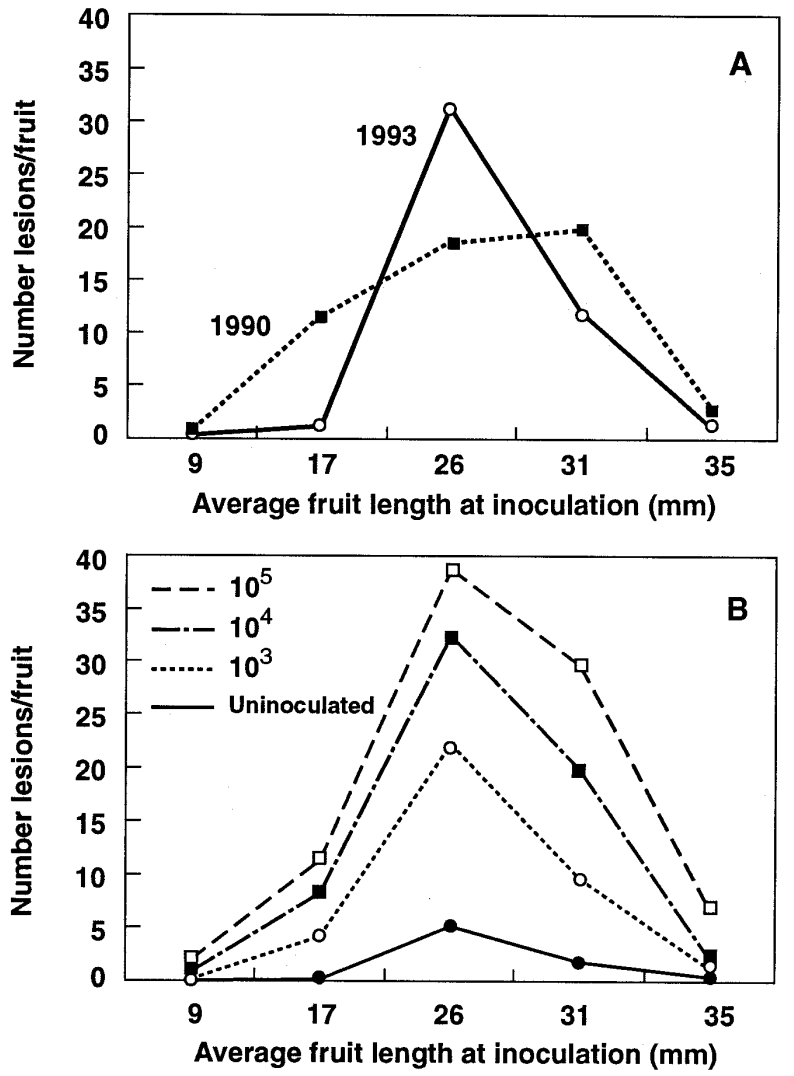

Fig. 2. Interactions of year (A) and inoculum concentration (B) with fruit length at inoculation for average number of fruit lesions on Mission almond fruit inoculated with Wilsonomyces carpophilus, Fresno County, California. Sixty fruit in each of four replications were inoculated during March and April 1990 and 1993 with 0.5 to $1.0 \mathrm{ml}$ (greater volume was required to cover larger fruit) of aqueous suspensions of $10^{3}, 10^{4}$, or $10^{5}$ conidia/ml, or left uninoculated. Average fruit length at inoculation was determined from 20 fruit selected arbitrarily from among inoculated fruit and measured on each inoculation date. The number of lesions was counted on 10 fruit per replication on 29 May 1990 and 15 June 1993. 
year was $17.3,53.3,9.1$ and $31.2 \mathrm{~mm}$ in 1990, 1993, 1994, and 1995, respectively, and during the inoculation periods each year was 24.1, 76.4, 40.4, and $20.3 \mathrm{~mm}$ in 1990, 1993, 1994, and 1995, respectively. In 1990, the average daily maximum temperatures during 1 to 31 March and 1 to 21 April were 22.4 and $24.5^{\circ} \mathrm{C}$, respectively, whereas in 1993 the temperatures during these periods were 21.0 and $21.9^{\circ} \mathrm{C}$.

\section{DISCUSSION}

Almond fruit appear to go through three stages of susceptibility to shot hole. Very young infected fruit are apt to abscise from the tree, and hull lesions are scarce on those that remain. Infected mid-size fruit also drop, but surviving fruit exhibit typical hull lesions. When fruit approach full size and embryo development begins, they become almost resistant to infection; abscission ceases and few hull lesions form.

It is difficult to assess with confidence the response of very young fruit to infection because the majority of both inoculated and uninoculated fruit that were present shortly after bloom eventually abscised as a result of poor pollination or natural thinning. Increases in abscission and lesion numbers were associated with increased inoculum concentration only when fruit of intermediate development were inoculated. Very young or full-size fruit did not vary in responses to inoculum concentration. Among the abscised young fruit we collected in April, typical discrete shot hole lesions were uncommon. Instead, areas of coalesced lesions or no obvious shot hole symptoms usually were observed. Apparently, most of the typical shot hole lesions observed on almond fruit result from infection of fruit intermediate in development. The cause of fruit abscission was not readily apparent because visible infections were not aggregated near the attachment to the pedicel.

Shaw et al. (8) demonstrated that abscission of shot hole lesions from almond leaves was influenced by temperature but not by the duration of wetness periods. Leaf lesions abscised most frequently at $22^{\circ} \mathrm{C}$, but seldom at 8 or $15^{\circ} \mathrm{C}$, and longer wetness periods promoted more lesions but not more lesion abscission. Adaskaveg (1) later documented the formation of abscission zones around lesions on leaves held at $22^{\circ} \mathrm{C}$, but not at $15^{\circ} \mathrm{C}$. During our studies, the average daily maximum ambient temperature was somewhat higher in 1990 than in 1993 ( 1.4 to $2.5^{\circ} \mathrm{C}$ difference between years), but fruit abscission was not correspondingly greater in 1990 . The magnitude of temperature differences during our experiments may have been insufficient to induce variance in the amount of abscission, or the environmental conditions that generate abscission of fruit and leaves may differ from those which cause lesion abscission. Although shot hole lesions developed on leaves close to our inoculated fruit, leaf loss was not observed in any of our treatments.

The high percent abscission observed in 1993 among all treatments, including the uninoculated control, may have resulted from poor pollination. Almond trees are not self-fertile; thus, pollen carried by bees from the companion Nonpareil trees was required to set fruit on the Mission trees. Peak bloom of Nonpareil trees generally occurs earlier than that of Mission trees; therefore, these cultivars are not ideal pollenizers for each other (3). This, coupled with the greater amount of rain that fell during bloom in 1993, which may have interrupted bee flight and pollination, could account for the higher abscission rate among small fruit observed that year than in 1990. As fruit aged, selection of viable fruit was easier, and percent abscission among the test fruit lessened. In the kernel quality experiments on Carmel trees, the higher average number of lesions per fruit found in 1994 perhaps reflects the greater amount of rain that fell during the inoculation period that year than in 1995 .
Fruit abscission clearly is a component of yield loss. As mentioned earlier, defoliation can trigger abscission of healthy and diseased fruit $(4,5,7)$. This response, along with the loss of tree vigor and fruiting wood that follow repeated defoliation, has been viewed as the basis for yield loss from shot hole disease. Our experiments show that infected young fruit also may fall independently of defoliation. In addition to yield reduction sustained as a consequence of defoliation, fruit may be lost directly to infection without substantial defoliation.

The effect of shot hole infection on kernel quality was negligible. Even multiple infections and coalesced lesions, more severe than often found in commercial orchards, did not reduce kernel weight or cause excessive shriveling or lacquering of the kernels. Cultivars that are more prone to gumming might produce more lacquered kernels in response to shot hole infection. The slight $(0.5 \mathrm{~mm})$ reduction in average kernel length found among inoculated fruit is not of economic importance. It does, however, suggest that kernel quality may
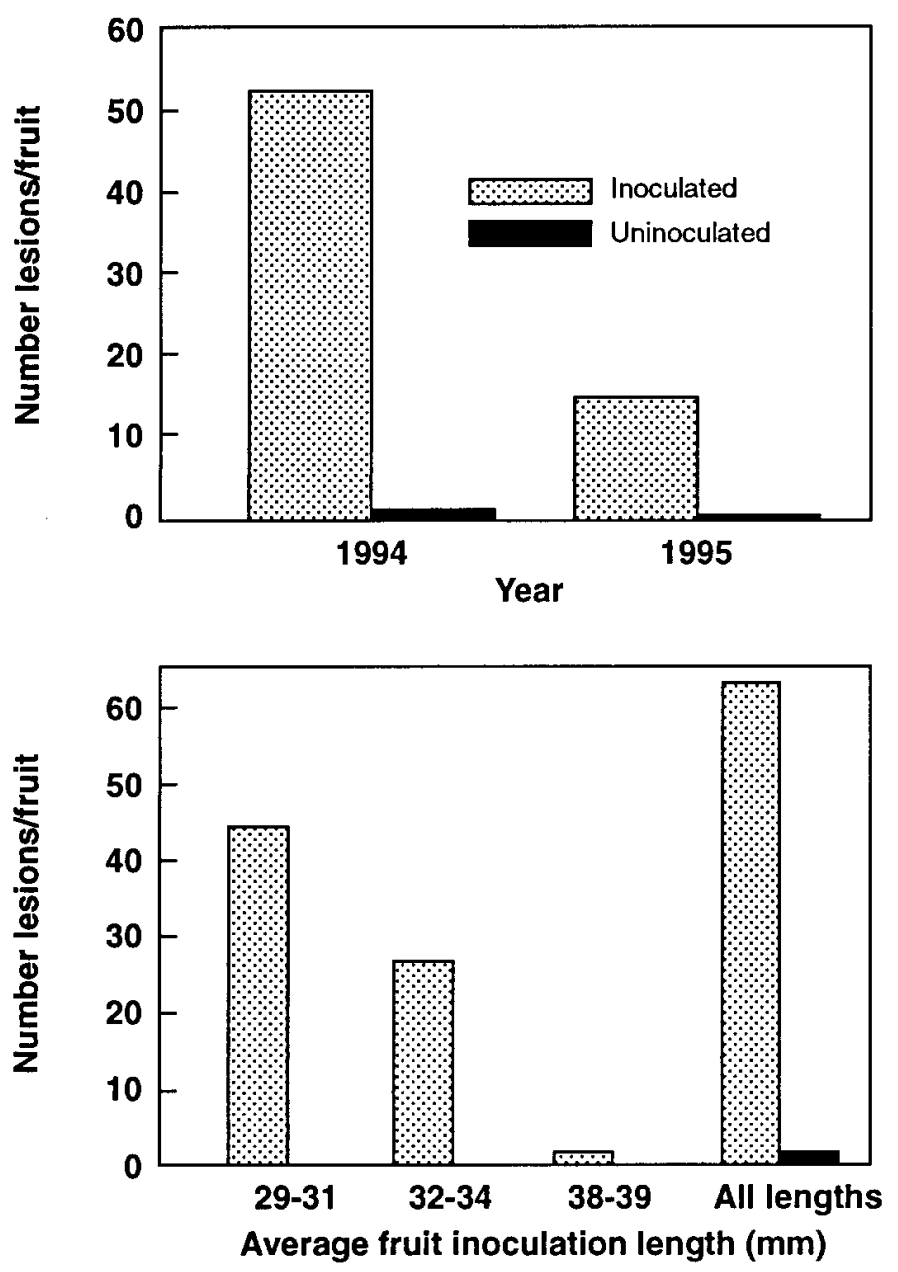

Fig. 3. Interactions of year (A) and inoculum concentration (B) with fruit length at inoculation for infection of Carmel almond fruit inoculated with Wilsonomyces carpophilus, Fresno County, California. Fruit were inoculated with $10^{5}$ conidia/ml or left uninoculated, and lesions were counted on 10 fruit in each of four replications on 23 June 1994 and 6 July 1995. Fifty fruit per replication were harvested on 29 and 26 August 1994 and 1995, respectively. 
Table 2. Effect of shot hole disease on kernel quality of Carmel almond fruit inoculated with Wilsonomyces carpophilus, Fresno County, California

\begin{tabular}{|c|c|c|c|}
\hline & Weight $(g)^{a}$ & Length $(\mathbf{m m})^{a}$ & Lesions/fruit ${ }^{\mathbf{b}}$ \\
\hline \multicolumn{4}{|l|}{ Year } \\
\hline 1994 & 1.21 & 26.5 & 27.1 \\
\hline 1995 & 1.12 & 25.2 & 7.7 \\
\hline \multicolumn{4}{|l|}{ Inoculation $^{\mathrm{d}}$} \\
\hline Inoculated & 1.11 & 25.6 & 34.4 \\
\hline Uninoculated & 1.12 & 26.1 & 0.5 \\
\hline \multicolumn{4}{|c|}{ Average fruit length at inoculation $(\mathrm{mm})^{\mathrm{c}}$} \\
\hline $29-31$ & 1.17 & 25.6 & 22.3 \\
\hline $32-34$ & 1.15 & 25.8 & 13.5 \\
\hline $38-39$ & 1.14 & 26.2 & 0.9 \\
\hline All lengths & 1.18 & 25.9 & 33.9 \\
\hline \multicolumn{4}{|l|}{ Significance of $F, P={ }^{\mathrm{d}}$} \\
\hline Year $(\mathrm{Y})$ & 0.002 & 0.001 & 0.001 \\
\hline Inoculation (I) & NS & 0.007 & 0.001 \\
\hline Fruit length at inoculation (L) & NS & NS & 0.001 \\
\hline $\mathrm{Y} \times \mathrm{I}$ & NS & NS & 0.001 \\
\hline $\mathrm{Y} \times \mathrm{L}$ & NS & NS & NS \\
\hline $\mathrm{I} \times \mathrm{L}$ & NS & NS & 0.001 \\
\hline \multicolumn{4}{|l|}{ Orthogonal contrasts } \\
\hline \multicolumn{4}{|l|}{ Fruit length at inoculation } \\
\hline Linear & $\ldots$ & $\ldots$ & 0.001 \\
\hline Single vs. multiple inoculation & NS & NS & 0.001 \\
\hline
\end{tabular}

${ }^{\text {a }}$ Fifty fruit per replication harvested on 29 and 26 August 1994 and 1995, respectively.

b Ten fruit per replication observed on 23 June 1994 and 6 July 1995.

${ }^{\mathrm{c}}$ Each of 70 fruit per replication were inoculated once with approximately 0.7 to $1.0 \mathrm{ml}$ of an aqueous suspension of $10^{5}$ conidia per $\mathrm{ml}$ on each inoculation date. The length $(\mathrm{mm})$ of 20 fruit, selected arbitrarily from among inoculated fruit, was measured on each inoculation date.

${ }^{\mathrm{d}}$ Four replications of each treatment. Means are for main effects; NS $=$ not significant, $P>0.010$.

be adversely affected by shot hole disease under some circumstances.

Leaves and fruit of almond trees are most likely to abscise if infected when young (10). When fruit are immature, many leaves on the tree also are young. In California, this stage of development coincides with the prolonged spring rains that favor shot hole. Infection by W. carpophilus at this time can result in defoliation and heavy fruit loss. Thus, it is important to protect almond trees from shot hole infection during this susceptible period.

\section{ACKNOWLEDGMENTS}

We thank Valeshia Hines, Don Self, Andrew Nunez, Tom Turini, and Andrea Chiamori for technical assistance, and the Almond Board of California for financial support for this research

LITERATURE CITED

1. Adaskaveg, J. E. 1995. Conidial morphology, host colonization, and development of shot hole of almond caused by Wilsonomyces carpophilus. Can. J. Bot. 73:432-444.

2. Aldrich, T., Moller, W. J., and Schulback, H. 1974. Shot hole disease control in almondsby injecting fungicides into overhead sprinklers. Calif. Agric. 28:11.

3. Asai, W. K., Micke, W. C., Kester, D. E., and Rough, D. 1996. The evaluation and selection of current varieties. Pages 52-60 in: Almond Production Manual. Div. Agric. Nat. Resources, Univ. Calif. Publ. 3364.

4. Highberg, L. M., and Ogawa, J. M. 1986 Yield reduction in almond related to incidence of shot hole disease. Plant Dis. 70:825-828.

5. Kester, D. E. 1996. Growth and development Pages 90-97 in: Almond Production Manual. Div. Agric. Nat. Resources, Univ. Calif. Publ. 3364

6. Ogawa, J. M., and English, H. 1991. Shot hole of stone fruit. Pages 193-196 in: Diseases of Temperate Zone Tree Fruit and Nut Crops. Div. Agric. Nat. Resources, Univ. Calif. Publ. 3345.

7. Ross, N. 1976. Pests and diseases. Pages 14.0 to $14.8 \mathrm{~b}$ in: Stanislaus Orchard Handbook. Univ. Calif. Cooperative Extension, Modesto, California.

8. Shaw, D. A., Adaskaveg, J. E., and Ogawa, J. M. 1990. Influence of wetness period and temperature on infection and development of shot-hole disease of almond caused by Wilsonomyces carpophilus, Phytopathology 80: 749-756.

9. Teviotdale, B. L., Viveros, M., Freeman, M. W., and Sibbett, G. S. 1989. Effect of fungicides on shot hole disease of almonds. Calif. Agric. 43:21-23.

10. Wilson, E. E. 1937. The shot hole disease of stone fruit trees. Univ. Calif. Agric. Exp. Stn. Bull. 608:3-40. 